Pacific Journal of Mathematic 


\title{
SEQUENCES OF CONTRACTIONS AND FIXED POINTS
}

\author{
SAM B. NADLER, JR.
}

Given a convergent sequence of contraction mappings, the convergence of the sequence of their fixed points is investigated in $\S 1$ of this paper. The results obtained lead to a necessary and sufficient condition in order that a separable or a reflexive Banach space be finite dimensional. An application to differential equations is also included.

In $\S 2$ we consider mappings defined on the cartesian product of two metric spaces which are contraction mappings in one variable or in each variable separately. Using some of the results of $\S 1$ we prove that, with certain restrictions, such mappings have fixed point.

Let $(X, d)$ be a metric space. A function $A: X \rightarrow X$ is said to be a contraction mapping if and only if there is a real number $\alpha$, $0 \leqq \alpha<1$, such that $d(A(x), A(y)) \leqq \alpha d(x, y)$ for all $x, y \in X$. The contraction mapping principle of Banach guarantees a unique fixed point of each contraction mapping of a complete metric space into itself. A natural question to ask is the following: In a complete metric space does the convergence of a sequence of contraction mappings to a contraction mapping $A_{0}$ imply the convergence of the sequence of their fixed points to the fixed point of $A_{0}$ ? A partial answer to this question appears as Theorem 1.2 of $[1, \mathrm{p} .6]$ ("Let $E$ be a complete metric space, and let $T$ and $T_{n}(n=1,2, \cdots)$ be contraction mappings of $E$ into itself with the same Lipschitz constant $K<1$, and with fixed points $u$ and $u_{n}$ respectively. Suppose that $\lim _{n \rightarrow \infty} T_{n}(x)=T(x)$ for every $x \in E$. Then $\lim _{n \rightarrow \infty} u_{n}=u$.") The restriction in this theorem, that all the contraction mappings have the "same Lipschitz constant $K<1$ ", is very strong; for one can easily construct a sequence of contraction mappings from the reals into the reals which converges uniformly to the zero mapping but whose Lipschitz constants tend to one.

In the next section two types of convergence of contraction mappings are considered uniform convergence and pointwise convergence. The question posed above is answered affirmatively in the case of uniform convergence and in the case of pointwise convergence on locally compact spaces.

1. Continuity of fixed points. We prove the following two main theorems:

Theorem 1. Let $(X, d)$ be a metric space, let $A_{i}: X \rightarrow X$ be a 
function with at least one fixed point $a_{i}$ for each $i=1,2, \cdots$, and let $A_{0}: X \rightarrow X$ be a contraction mapping with fixed point $a_{0}$. If the sequence $\left\{A_{i}\right\}_{i=1}^{\infty}$ converges uniformly to $A_{0}$, then the sequence $\left\{a_{i}\right\}_{i=1}^{\infty}$ converges to $a_{0}$.

THEOREM 2. Let $(X, d)$ be a locally compact metric space, let $A_{i}: X \rightarrow X$ be a contraction mapping with fixed point $a_{i}$ for each $i=$ $1,2, \cdots$, and let $A_{0}: X \rightarrow X$ be a contraction mapping with fixed point $a_{0}$. If the sequence $\left\{A_{i}\right\}_{i=1}^{\infty}$ converges pointwise to $A_{0}$, then the sequence $\left\{a_{i}\right\}_{i=1}^{\infty}$ converges to $a_{0}$.

Proof of Theorem 1. Let $\varepsilon>0$ and choose a natural number $N$ such that $i \geqq N$ implies $d\left(A_{i}(x), A_{0}(x)\right)<\varepsilon\left(1-\alpha_{0}\right)$ for all $x \in X$, where $\alpha_{0}<1$ is a Lipschitz constant for $A_{0}$. Then, if

$$
\begin{aligned}
i \geqq & N, d\left(a_{i}, a_{0}\right)=d\left(A_{i}\left(a_{i}\right), A_{0}\left(a_{0}\right)\right) \leqq d\left(A_{i}\left(a_{i}\right), A_{0}\left(a_{i}\right)\right) \\
& +d\left(A_{0}\left(a_{i}\right), A_{0}\left(a_{0}\right)\right)<\varepsilon\left(1-\alpha_{0}\right)+\alpha_{0} d\left(a_{i}, a_{0}\right) .
\end{aligned}
$$

Hence, $d\left(a_{i}, a_{0}\right)<\varepsilon$ for all $i \geqq N$. This proves that $\left\{a_{i}\right\}_{i=1}^{\infty}$ converges to $a_{0}$ and completes the proof of Theorem 1 .

Proof of Theorem 2. Let $\varepsilon>0$ and assume $\varepsilon$ is sufficiently small so that $K\left(a_{0}, \varepsilon\right)=\left\{x \in X \mid d\left(a_{0}, x\right) \leqq \varepsilon\right\}$ is a compact subset of $X$. Then, since $\left\{A_{i}\right\}_{i=1}^{\infty}$ is an equicontinuous sequence of functions converging pointwise to $A_{0}$ and since $K\left(a_{0}, \varepsilon\right)$ is compact, the sequence $\left\{A_{i}\right\}_{i=1}^{\infty}$ converges uniformly on $K\left(a_{0}, \varepsilon\right)$ to $A_{0}$. Choose $N$ such that if $i \geqq N$, then $d\left(A_{i}(x), A_{0}(x)\right)<\left(1-\alpha_{0}\right) \varepsilon$ for all $x \in K\left(a_{0}, \varepsilon\right)$, where $\alpha_{0}<1$ is a Lipschitz constant for $A_{0}$. Then, if $i \geqq N$ and $x \in K\left(a_{0}, \varepsilon\right), d\left(A_{i}(x), a_{0}\right)$ $\leqq d\left(A_{i}(x), A_{0}(x)\right)+d\left(A_{0}(x), A_{0}\left(a_{0}\right)\right)<\left(1-\alpha_{0}\right) \varepsilon+\alpha_{0} d\left(x, a_{0}\right) \leqq\left(1-\alpha_{0}\right) \varepsilon+$ $\alpha_{0} \varepsilon=\varepsilon$. This proves that if $i \geqq N$, then $A_{i}$ maps $K\left(a_{0}, \varepsilon\right)$ into itself. Letting $B_{i}$ be the restriction of $A_{i}$ to $K\left(a_{0}, \varepsilon\right)$ for each $i \geqq N$ we see that each $B_{i}$ is a contraction mapping of $K\left(a_{0}, \varepsilon\right)$ into itself. Since $K\left(a_{0}, \varepsilon\right)$ is a complete metric space, $B_{i}$ has a fixed point for each $i \geqq N$ which must, from the definition of $B_{i}$ and the fact that $A_{i}$ has only one fixed point, be $a_{i}$. Hence, $a_{i} \in K\left(a_{0}, \varepsilon\right)$ for each $i \geqq N$. It follows that the sequence $\left\{a_{i}\right\}_{i=1}^{\infty}$ of fixed points converges to $a_{0}$. This completes the proof of Theorem 2 .

We now give an example which shows that, in non-locally compact spaces, a sequence of contraction mappings may converge pointwise to a contraction mapping without the sequence of their fixed points converging. In fact, the example is a construction in any infinite dimensional separable or reflexive Banach space of a sequence of contraction mappings which converges pointwise to the zero mapping but such 
that the sequence of their fixed points has no convergent subsequence. The following lemma will be useful in this example.

Lemma. Let $(X, d)$ be a metric space, let $A_{i}: X \rightarrow X$ be a contraction mapping with fixed point $a_{i}$ for each $i=1,2, \cdots$, and let $A_{0}: X \rightarrow X$ be a contraction mapping with fixed point $a_{0}$. If the sequence $\left\{A_{i}\right\}_{i=1}^{\infty}$ converges pointwise to $A_{0}$ and if a subsequence $\left\{a_{i_{j}}\right\}_{j=1}^{\infty}$ of $\left\{a_{i}\right\}_{i=1}^{\infty}$ converges to a point $x_{0} \in X$, then $x_{0}=a_{0}$.

Proof. Let $\varepsilon>0$. Then there is a positive integer $N$ such that $j \geqq N$ implies $d\left(a_{i_{j}}, x_{0}\right)<(\varepsilon / 2)$ and $d\left(A_{i_{j}}\left(x_{0}\right), A_{0}\left(x_{0}\right)\right)<(\varepsilon / 2)$. Therefore, $d\left(a_{i_{j}}, A_{0}\left(x_{0}\right)\right)=d\left(A_{i_{j}}\left(a_{i_{j}}\right), A_{0}\left(x_{0}\right)\right) \leqq d\left(A_{i_{j}}\left(a_{i_{j}}\right), A_{i_{j}}\left(x_{0}\right)\right)+d\left(A_{i_{j}}\left(x_{0}\right), A_{0}\left(x_{0}\right)\right)<$ $d\left(a_{i_{j}}, x_{0}\right)+d\left(A_{i_{j}}\left(x_{0}\right), A_{0}\left(x_{0}\right)\right)<\varepsilon$ for all $j \geqq N$. This proves that the sequence $\left\{a_{i_{j}}\right\}_{j=1}^{\infty}$ converges to $A_{0}\left(x_{0}\right)$. Hence, $A_{0}\left(x_{0}\right)=x_{0}$ and it follows that $x_{0}=a_{0}$.

ExAmple 1. Let $B$ be an infinite dimensional separable or reflexive Banach space. Let $B^{*}$ be the first conjugate of $B$ and let $T=$ $\left\{f \in B^{*} \mid\|f\| \leqq 1\right\}$. Then $T$ is weak* sequentially compact. In the separable case this follows from the metrizability of the weak* topology for $T[2, \mathrm{p} .426]$; in the reflexive case the weak compactness of $T$ implies, by the Eberlein-Smulian Theorem [2, p. 430], that $T$ is weakly sequentially compact and, therefore, weak* sequentially compact. Since $B$ is infinite dimensional, there is a sequence $\left\{g_{k}\right\}_{k=1}^{\infty}$ of linear functionals in $T$ which has no norm convergent subsequence. Let $\left\{g_{k_{i}}\right\}_{i=1}^{\infty}$ be a weak* convergent subsequence of $\left\{g_{k}\right\}_{k=1}^{\infty}$ and let $g$ be the weak* limit of $\left\{g_{k_{i}}\right\}_{i=1}^{\infty}$. For each $i=1,2, \cdots$ let

$$
f_{i}=\frac{g_{k_{i}}-g}{\left\|g_{k_{i}}-g\right\|} .
$$

The sequence $\left\{f_{i}\right\}_{i=1}^{\infty}$ is weak ${ }^{*}$ convergent to the zero linear functional and $\left\|f_{i}\right\|=1$ for all $i=1,2, \cdots$.

For each $i=1,2, \cdots$, let $a_{i} \in B$ such that $\left\|a_{i}\right\|=1$ and $\left|f_{i}\left(a_{i}\right)\right|$ $>1-\left(1 / i^{2}\right)$. For each $i=1,2, \cdots$, define $A_{i}: B \rightarrow B$ by

$$
A_{i}(x)=\left(1-\frac{1}{i}\right) \frac{f_{i}(x)}{f_{i}\left(a_{i}\right)} a_{i}+\frac{1}{i} a_{i}
$$

for all $x \in B$. Since

$$
\begin{aligned}
& \left\|A_{i}(x)-A_{i}(y)\right\|=\|\left(1-\frac{1}{i}\right) \frac{f_{i}(x)}{f_{i}\left(a_{i}\right)} a_{i} \\
& \quad-\left(1-\frac{1}{i}\right) \frac{f_{i}(y)}{f_{i}\left(a_{i}\right)} a_{i}\left\|=\left(1-\frac{1}{i}\right) \frac{\left|f_{i}(x)-f_{i}(y)\right|}{\left|f_{i}\left(a_{i}\right)\right|}\right\| a_{i} \|
\end{aligned}
$$




$$
\leqq\left(1-\frac{1}{i}\right) \frac{\|x-y\|}{\left|f_{i}\left(a_{i}\right)\right|} \leqq \frac{i}{1+i}\|x-y\|
$$

for all $x$ and $y$ in $B, A_{i}$ is a contraction mapping for each $i=1,2, \cdots$. Since the sequence $\left\{f_{i}\right\}_{i=1}^{\infty}$ is weak* convergent to the zero linear functional and the sequence $\left\{f_{i}\left(a_{i}\right)\right\}_{i=1}^{\infty}$ is bounded away from zero, it follows that the sequence $\left\{A_{i}\right\}_{i=1}^{\infty}$ converges pointwise to the zero mapping. It is easy to verify that, for each $i=1,2, \cdots, a_{i}$ is the unique fixed point of $A_{i}$. Since $\left\|a_{i}\right\|=1$ for all $i=1,2, \cdots$, it follows from the lemma that the sequence $\left\{a_{i}\right\}_{i=1}^{\infty}$ of fixed points has no convergent subsequence. Hence, $\left\{A_{i}\right\}_{i=1}^{\infty}$ is a sequence of contraction mappings which converges pointwise to the zero mapping and such that the sequence $\left\{a_{i}\right\}_{i=1}^{\infty}$ of fixed points has no convergent subsequence. This example may be slightly modified so that the sequence of fixed points is unbounded [5]. Also it is clear that this type of construction may be done in any infinite dimensional Banach space in which the unit ball of the first conjugate is weak* sequentially compact.

The next theorem is a characterization of those separable Banach spaces which are finite dimensional in terms of pointwise convergent sequences of contraction mappings and the convergence of their fixed points.

Theorem 3. A separable or reflexive Banach space $B$ is finite dimensional if and only if whenever a sequence of contraction mappings of $B$ into $B$ converges pointwise to a contraction mapping $A_{0}$, then the sequence of their fixed points converges to the fixed point of $A_{0}$.

Proof. Since a finite dimensional Banach space is locally compact, half of the theorem follows from Theorem 2. The proof of the other half of the theorem is obtained by supposing $B$ is not finite dimensional and applying Example 1.

It is not known by the author whether or not the statement of Theorem 3 remains valid if the condition, "separable or reflexive," is removed.

As an application of Theorem 2 we give the following proposition due to Professor J. R. Dorroh.

Proposition. Let $D$ be an open subset of the plane, let $(a, b) \in D$, let $M>0$ be a real number, and let $\left\{K_{i}\right\}_{i=0}^{\infty}$ be a bounded sequence of strictly positive real numbrs. For each $i=0,1,2, \cdots$, let $f_{i}$ be a real valued continuous function defined on $D$ such that $\left|f_{i}(x, y)\right| \leqq M$ for all $(x, y) \in D$ and $\left|f_{i}(x, y)-f_{i}(x, z)\right| \leqq K_{i}|y-z|$ for all $(x, y),(x, z) \in D$. 
Suppose also that the sequence $\left\{f_{i}\right\}_{i=1}^{\infty}$ converges pointwise on $D$ to $f_{0}$. Let $h$ be such that $0<k_{i} \cdot h<1$ for all $i=0,1,2, \cdots$, and such that the set $W=\{(x, y)|| x-a \mid \leqq h$ and $|y-b| \leqq M|x-a|\}$ is a subset of $D$. Then the sequence $\left\{y_{i}\right\}_{2=1}^{\infty}$ converges uniformly on $I=[a-h, a+h]$ to $y_{0}$ where, for each $i=0,1,2, \cdots, y_{i}$ is the unique solution on $I$ of the initial value problem

$$
\begin{aligned}
& y(a)=b \\
& y^{\prime}(x)=f_{i}(x, y(x)) .
\end{aligned}
$$

Proof. Let $X$ be the set of all real valued functions defined on $I$ with graph lying in $W$ and with Lipschitz constant less than or equal to $M$. Then $X$, with the supremum metric $\rho$, is a compact metric space. For each $i=0,1,2, \cdots$ and each $g \in X$, define $A_{i}(g)$ at each $x \in I$ by $\left[A_{i}(g)\right](x)=b+\int_{a}^{x} f_{i}(t, g(t)) d t$. It is easy to verify that, for each $i=0,1,2, \cdots, A_{i}$ is a contraction mapping from $X$ into $X$ with Lipschitz constant less than or equal to $K_{i} \cdot h$. For each $g \in X$, $x \in I$, and $i=1,2, \cdots$,

$$
\left[A_{i}(g)\right](x)-\left[A_{0}(g)\right](x)=\int_{a}^{x}\left[f_{i}(t, g(t))-f_{0}(t, g(t))\right] d t .
$$

Since the sequence of integrands converges pointwise to zero and is uniformly bounded by $2 M$, the Lebesgue bounded convergence theorem guarantees that the sequence of integrals goes to zero as $i$ tends to infinity. Therefore, the sequence $\left\{A_{i}(g)\right\}_{i=1}^{\infty}$ converges pointwise on $I$ to $A_{0}(g)$. This implies, by the equicontinuity of $\left\{A_{i}(g)\right\}_{i=1}^{\infty}$ on the compact set 1 , that the sequence $\left\{A_{i}(g)\right\}_{i=1}^{\infty}$ converges uniformly on $I$ to $A_{0}(g)$. Hence, the sequence $\left\{A_{i}\right\}_{i=1}^{\infty}$ converges pointwise on $X$ to $A_{0}$. By Theorem 2 the sequence $\left\{y_{i}\right\}_{i=1}^{\infty}$, where $y_{i}$ is the unique fixed point of $A_{i}$ for each $i=1,2, \cdots$, converges to the fixed point $y_{0}$ of $A_{0}$. The result follows since these fixed points are the unique solutions of the initial value problem.

The restriction in this proposition that each of the mappings $f_{1}$, $f_{2}, \cdots$ satisfy the type of Lipschitz condition given above can be significantly weakened. This and related matters will be considered for a later article.

2. A fixed point theorem for product spaces. A number of mathematicians have investigated the problem of determining what kinds of mappings defined on the cartesian product of two spaces have fixed points (for an historical survey see [6]). In 1930, K. Kuratowski asked [3] if the cartesian product of two Peano continua, each with the fixed point property, had the fixed point property. Recently, W. 
Lopez [4] gave an example of a finite polyhedron with the fixed point property whose cartesian product with the unit interval failed to have the fixed point property.

Throughout this section $\left(X, d_{X}\right)$ and $\left(Y, d_{Y}\right)$ will be metric spaces and $(X \times Y, d)$ will denote their cartesian product with the product metric $d$ given by $d\left(\left(x_{1}, y_{1}\right),\left(x_{2}, y_{2}\right)\right)=\left[\left(d_{X}\left(x_{1}, x_{2}\right)\right)^{2}+\left(d_{r}\left(y_{1}, y_{2}\right)\right)^{2}\right]^{1 / 2}$ for all $\left(x_{1}, y_{1}\right),\left(x_{2}, y_{2}\right) \in X \times Y$. A function $f: X \times Y \rightarrow X \times Y$ is said to be a contraction mapping in the first variable if and only if for each $y \in Y$ there is a real number $\alpha(y), 0 \leqq \alpha(y)<1$, such that $d\left(f\left(x_{1}, y\right)\right.$, $\left.f\left(x_{2}, y\right)\right) \leqq \alpha(y) d\left(\left(x_{1}, y\right),\left(x_{2}, y\right)\right)$ for all $x_{1}, x_{2} \in x$. We define a contraction mapping in the second variable in an analogous fashion and we say that a function is a contraction mapping in each variable separately provided it is a contraction mapping in the first variable and in the second variable.

It is worthwhile noting that, even if $\left(X, d_{X}\right)$ and $\left(Y, d_{Y}\right)$ are compact, there may be mappings from $X \times Y$ into $X \times Y$ which are contraction mappings in each variable separately but which are not themselves contraction mappings. The function $f:[0,1] \times[0,1] \rightarrow[0,1]$ $\times[0,1]$ given by $f(x, y)=\{(x+y) / 2,(x+y) / 2\}$ for all $(x, y) \in[0,1] \times[0,1]$ is an example of such a mapping.

THeorem 4. Let $\left(X, d_{X}\right)$ be a complete metric space, let $\left(Y, d_{Y}\right)$ be a metric space with the fixed point property, and let $f$ be a function from $X \times Y$ into $X \times Y$.

(1) If $f$ is uniformly continuous on $X \times Y$ and a contraction mapping in the first variable, then $f$ has a fixed point.

(2) If $\left(X, d_{X}\right)$ is locally compact and $f$ is continuous on $X \times Y$ and a contraction mapping in the first variable, then $f$ has a fixed point.

Proof. We prove (1) and (2) simultaneously. If $y \in Y$, then let $f_{y}: X \rightarrow X$ be defined by $f_{y}(x)=\pi_{1} \circ f(x, y)$ for all $x \in X$ where $\pi_{1}$ is the natural projection of $X \times Y$ onto $X$. For each $y \in Y, f_{y}$ is a contraction mapping of $X$ into $X$ and, therefore, has one and only one fixed point. Let $F: Y \rightarrow X$ be given by $F(y)$ is the unique fixed point of $f_{y}$. Now let $y_{0} \in Y$ and let $\left\{y_{i}\right\}_{i=1}^{\infty}$ be a sequence of points of $Y$ which converges to $y_{0}$. Under the assumption of 1 , the sequence $\left\{f_{y_{i}}\right\}_{i=1}^{\infty}$ converges uniformly to $f_{y_{0}}$ and hence, by Theorem 1 , the sequence $\left\{F\left(y_{i}\right)\right\}_{i=1}^{\infty}$ converges to $F\left(y_{0}\right)$. Under the assumptions of 2 , we may apply Theorem 2 to conclude that the sequence $\left\{F\left(y_{i}\right)\right\}_{i=1}^{\infty}$ converges to $F\left(y_{0}\right)$. Hence, in either case, this proves that $F$ is continuous on $Y$. Next let $G: Y \rightarrow Y$ be the continuous function defined by $G(y)=\pi_{2} \circ f(F(y), y)$ for each $y \in Y$ where $\pi_{2}$ is the natural projection of $X \times Y$ onto $Y$. Since $Y$ has the fixed point property, there is a 
point $p \in Y$ such that $G(p)=p$. It follows that $(F(p), p)$ is a fixed point of $f$, which proves Theorem 4 .

A special class of functions satisfying the conditions of (1) are Lipschitz functions which are contraction mappings in the first variable. Theorem 4, therefore, gives a class of Lipschitz mappings of $X \times Y$ into $X \times Y$ which have fixed points. Along these lines we have the following:

Corollary. Let $\left(X, d_{X}\right)$ be a complete metric space and let $(Y$, $d_{Y}$ ) be a metric space with the fixed point property. If $f: X \times Y \rightarrow$ $X \times Y$ is a contraction mapping in each variable separately, then $f$ has a fixed point.

It may seem that the type of restriction placed on $f$ in the corollary above would enable us to replace the condition " $\left(Y, d_{Y}\right)$ has the fixed point property" by the condition " $\left(Y, d_{Y}\right)$ is complete." However, the function $f: R^{1} \times R^{1} \rightarrow R^{1} \times R^{1}$ (where $R^{1}$ denotes the real numbers) defined by $f(x, y)=\{(x+y) / 2+1,(x+y) / 2+1)\}$ for all $(x, y) \in R^{1} \times$ $R^{1}$ shows that this is not the case. For it is easy to see that $f$ is a contraction mapping in each variable separately and has no fixed point.

REMARK: It is clear from the proof of Theorem 4 that less restrictive topological conditions could have been assumed about $Y$ (for example, in part 2, $Y$ need only be first axiom with the fixed point property).

The author wishes to express his appreciation to the referee for his valuable suggestions concerning the material in this paper.

\section{REFERENCES}

1. F. F. Bonsall, Lectures on Some Fixed Point Theorems of Functional Analysis, Tata Institute of Fundamental Research, Bombay, India, 1962.

2. N. Dunford and J. T. Schwartz, Linear Operators, Interscience Publishers Inc., New York, 1958.

3. K. Kuratowski, Problem 49, Fund. Math. 15 (1930), 356.

4. W. Lopez, An example in the fixed point theory of polyhedra, Bull. Amer. Math. Soc. 73 (1967), 922-924.

5. S. B. Nadler, Jr., A note on sequences of contractions, Proceedings of the Clemson Conference on Projections and Related Topics (1968), Department of Mathematics, Clemson University, Clemson, South Carolina.

6. T. Van Der Walt, Fixed and Almost Fixed Points, Mathematisch Centrum, Amsterdam, Holland, 1963.

Received February 9, 1968, and in revised form March 22, 1968. 



\section{PACIFIC JOURNAL OF MATHEMATICS}

\section{EDITORS}

\section{H. ROYDEN}

Stanford University

Stanford, California

\section{J. Dugundu}

Department of Mathematics University of Southern California Los Angeles, California 90007

RICHARD ARENS

University of California Los Angeles, California 90024

ASSOCIATE EDITORS
E. F. Beckenbach
B. H. NEUMANN
F. WoLF
K. YosidA

\section{SUPPORTING INSTITUTIONS}

\author{
UNIVERSITY OF BRITISH COLUMBIA \\ CALIFORNIA INSTITUTE OF TECHNOLOGY \\ UNIVERSITY OF CALIFORNIA \\ MONTANA STATE UNIVERSITY \\ UNIVERSITY OF NEVADA \\ NEW MEXICO STATE UNIVERSITY \\ OREGON STATE UNIVERSITY \\ UNIVERSITY OF OREGON \\ OSAKA UNIVERSITY \\ UNIVERSITY OF SOUTHERN CALIFORNIA
}

STANFORD UNIVERSITY

UNIVERSITY OF TOKYO

UNIVERSITY OF UTAH

WASHINGTON STATE UNIVERSITY

UNIVERSITY OF WASHINGTON

AMERICAN MATHEMATICAL SOCIETY
CHEVRON RESEARCH CORPORATION
TRW SYSTEMS

AMERICAN MATHEMATICAL SOCIETY

TRW SYSTEMS

NAVAL WEAPONS CENTER

Mathematical papers intended for publication in the Pacific Journal of Mathematics should be in typed form or offset-reproduced, double spaced with large margins. Underline Greek letters in red, German in green, and script in blue. The first paragraph or two must be capable of being used separately as a synopsis of the entire paper. It should not contain references to the bibliography. Manuscripts, in duplicate if possible, may be sent to any one of the four editors. All other communications to the editors should be addressed to the managing editor, Richard Arens, University of California, Los Angeles, California 90024.

Each author of each article receives 50 reprints free of charge; additional copies may be obtained at cost in multiples of 50 .

The Pacific Journal of Mathematics is published monthly. Effective with Volume 16 the price per volume (3 numbers) is $\$ 8.00$; single issues, $\$ 3.00$. Special price for current issues to individual faculty members of supporting institutions and to individual members of the American Mathematical Society: $\$ 4.00$ per volume; single issues $\$ 1.50$. Back numbers are available.

Subscriptions, orders for back numbers, and changes of address should be sent to Pacific Journal of Mathematics, 103 Highland Boulevard, Berkeley 8, California.

Printed at Kokusai Bunken Insatsusha (International Academic Printing Co., Ltd.), 7-17, Fujimi 2-chome, Chiyoda-ku, Tokyo, Japan.

PUBLISHED BY PACIFIC JOURNAL OF MATHEMATICS, A NON-PROFIT CORPORATION

The Supporting Institutions listed above contribute to the cost of publication of this Journal, but they are not owners of publishers and have no responsibility for its content or policies. 


\section{Pacific Journal of Mathematics \\ Vol. 27, No. 3}

March, 1968

Charles A. Akemann, Invariant subspaces of $C(G) \ldots \ldots \ldots \ldots \ldots \ldots . \ldots 41$

Dan Amir and Zvi Ziegler, Generalized convexity cones and their duals ... . 425

Raymond Balbes, On ( $J, M, \mathrm{~m})$-extensions of order sums of distributive

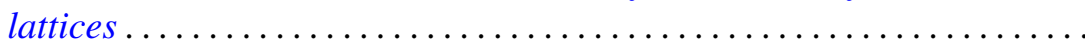

Jan-Erik Björk, Extensions of the maximal ideal space of a function algebra ........................................ 453

Frank Castagna, Sums of automorphisms of a primary abelian group ...... 463

Theodore Seio Chihara, On determinate Hamburger moment problems ..... .

Zeev Ditzian, Convolution transforms whose inversion function has complex

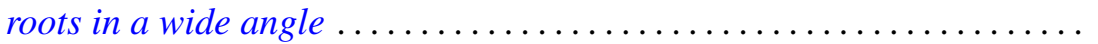

Myron Goldstein, On a paper of Rao .

Velmer B. Headley and Charles Andrew Swanson, Oscillation criteria for

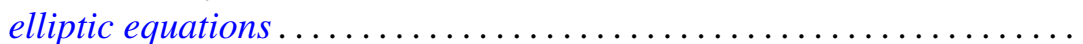

John Willard Heidel, Qualitative behavior of solutions of a third order nonlinear differential equation............................

Alan Carleton Hindmarsh, Pick's conditions and analyticity.............

Bruce Ansgar Jensen and Donald Wright Miller, Commutative semigroups

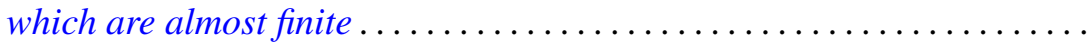

Lynn Clifford Kurtz and Don Harrell Tucker, An extended form of the

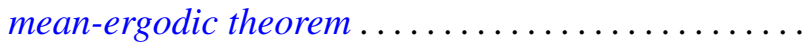

S. P. Lloyd, Feller boundary induced by a transition operator ...

Henry B. Mann, Josephine Mitchell and Lowell Schoenfeld, A new proof of the maximum principle for doubly-harmonic functions ...

Robert Einsohn Mosher, The product formula for the third obstruction ..

Sam Bernard Nadler, Jr., Sequences of contractions and fixed points ...

Eric Albert Nordgren, Invariant subspaces of a direct sum of weighted shifts...

Fred Richman, Thin abelian p-groups ...

Jordan Tobias Rosenbaum, Simultaneous interpolation in $\mathrm{H}_{2}$. II ...

Charles Thomas Scarborough, Minimal Urysohn spaces .

Malcolm Jay Sherman, Disjoint invariant subspaces..... .

Joel John Westman, Harmonic analysis on groupoids....

621

William Jennings Wickless, Quasi-isomorphism and TFM

Minoru Hasegawa, Correction to "On the convergence of resolvents of operators" 